\title{
The potential impact of animal protein intake on global and abdominal obesity: evidence from the Observation of Cardiovascular Risk Factors in Luxembourg (ORISCAV-LUX) study
}

\author{
Ala'a Alkerwi $^{1, *}$, Nicolas Sauvageot ${ }^{1}$, Jonathan D Buckley ${ }^{2}$, Anne-Françoise Donneau ${ }^{3}$, \\ Adelin Albert ${ }^{3}$, Michèle Guillaume ${ }^{3}$ and Georgina E Crichton ${ }^{1,2}$ \\ ${ }^{1}$ Centre de Recherche Public Santé, Centre d'Etudes en Santé, 1 A rue Thomas Edison, L-1445 Strassen, Luxembourg: \\ ${ }^{2}$ Nutritional Physiology Research Centre, University of South Australia, Adelaide, Australia: ${ }^{3}$ Département des \\ Sciences de la Santé Publique, Université de Liège, Liège, Belgium
}

Submitted 25 February 2014: Final revision received 15 September 2014: Accepted 1 0ctober 2014: First published online 22 January 2015

\begin{abstract}
Objective: To examine the association of total animal protein intake and protein derived from different dietary sources (meat; fish and shellfish; eggs; milk products) with global and abdominal obesity among adults in Luxembourg.

Design: Binary logistic regression analysis was used to assess the relationship between animal protein intake (as a percentage of total energy intake) and global obesity $\left(\mathrm{BMI} \geq 30 \cdot 0 \mathrm{~kg} / \mathrm{m}^{2}\right.$ ) and abdominal obesity (waist circumference $\geq 102 \mathrm{~cm}$ for men and $\geq 88 \mathrm{~cm}$ for women), after controlling for potential confounders. Setting: Observation of Cardiovascular Risk Factors in Luxembourg (ORISCAVLUX) study.

Subjects: The study population was derived from a national cross-sectional stratified sample of 1152 individuals aged 18-69 years, recruited between November 2007 and January 2009.

Results: There was an independent positive association between total animal protein intake and both global $(\mathrm{OR}=1 \cdot 18 ; 95 \% \mathrm{CI} 1 \cdot 12,1.25)$ and abdominal obesity $(\mathrm{OR}=1 \cdot 14 ; 95 \% \mathrm{CI} 1.08,1 \cdot 20)$ after adjustment for age, gender, education, smoking, physical activity and intakes of total fat, carbohydrate, fibre, and fruit and vegetables. Protein intakes from meat, fish and shellfish were positively associated with global and abdominal obesity with further adjustment for vegetal protein and other sources of animal-derived protein (all $P<0 \cdot 01$ ). Protein derived from eggs or milk products was unrelated to global or abdominal obesity.

Conclusions: Our findings suggest that protein derived from animal sources, in particular from meat, fish and shellfish, may be associated with increased risk of both global and abdominal obesity among presumably healthy adults in Luxembourg. These findings suggest that lower animal protein intakes may be important for maintenance of healthy body weight.
\end{abstract}

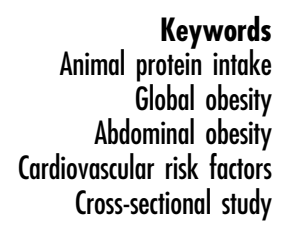

Obesity is a rapidly worsening public health problem associated with a variety of co-morbidities including type 2 diabetes, hypertension, stroke and $\mathrm{CVD}^{(1)}$. Obesity is impacting heavily upon health-care systems around the world, including Luxembourg, where $21 \%$ of the population is obese ${ }^{(2)}$. Obesity occurs in the context of a variety of interrelated demographic, socio-economic and lifestyle factors; nutrition is coming to the fore as a major modifiable determinant. Nutritional epidemiology has produced evidence that an energy-dense and high-fat diet, concomitant with physical inactivity, are independent risk factors for weight gain and obesity ${ }^{(3-5)}$.
The literature surrounding the effects of protein intake and particular sources of protein on body composition is unclear. Three recent meta-analyses have been conducted, comparing higher- $v$. lower-protein diets on health outcomes, including body composition ${ }^{(6-8)}$, with conflicting conclusions. One indicates that a high protein intake in the context of an energy-restricted diet provides greater improvement in body composition compared with standard protein diets, matched for energy intake ${ }^{(8)}$. Another systematic review concluded that higher-protein diets probably improve adiposity than lower-protein diets, but the effect is small ${ }^{(6)}$. The most recent meta-analysis, which 
included studies using both energy-restricted and nonenergy-restricted diets, reported no added benefit for body weight or body composition from a high-protein diet $v$. lower-protein diets ${ }^{(7)}$. The reason for these different findings is not clear, but suggests that higher protein intakes may only benefit body composition when consumed as part of a weight-loss diet as there is no convincing evidence linking dietary protein intake and body weight under conditions of weight maintenance ${ }^{(9)}$. Nevertheless, protein-rich diets could have potential beneficial effects by increasing satiety and thermogenesis ${ }^{(10)}$, and other studies have suggested that replacing carbohydrate with protein from meat, poultry and dairy foods may have beneficial metabolic effects $^{(11)}$ and help reduce abdominal obesity ${ }^{(12)}$.

Evidence is emerging that the type of dietary protein consumed can elicit different health effects and play an important role in disease aetiology ${ }^{(13)}$. In general, plant proteins have been related to health benefits more than animal proteins ${ }^{(14-17)}$. While vegetable protein intakes have been found to be inversely associated with blood pressure $^{(18)}$, a high consumption of red and/or processed meat has been associated with a number of adverse cardiovascular health outcomes such as higher systolic blood pressure ${ }^{(19)}$, increased risk for type 2 diabetes ${ }^{(20-22)}$, ischaemic stroke ${ }^{(23)}$, global and central obesity ${ }^{(24,25)}$ and weight gain $^{(26,27)}$.

Although the adverse effects of red and processed meat consumption are well documented, there is still lack of firm evidence regarding the effect of other sources of animal protein intake (fish, eggs and milk products) on health outcomes, including body weight status ${ }^{(9,28)}$. The present study aimed to examine the association of animal protein intake and more specifically, intakes of animal protein derived from different sources, i.e. meats (red meat, poultry), fish and shellfish, eggs and milk products, with global and abdominal obesity in a nationally representative sample of adult participants in the Observation of Cardiovascular Risk Factors in Luxembourg (ORISCAVLUX) survey in Luxembourg. It was hypothesized that animal protein would be positively associated with obesity, but the association may vary according to protein source. The findings will contribute to current knowledge on the influence of animal protein intake on body weight status and help guide the development of future prevention/weight control intervention studies.

\section{Methods}

\section{Study population}

The study material consisted of individuals from the national ORISCAV-LUX study, a cross-sectional, population-based survey among adults aged 18-69 years. The data collection, sample design and representativeness have been reported in detail elsewhere ${ }^{(2,29,30)}$. Briefly, a stratified random sample of 1432 non-institutionalized individuals was enrolled between November 2007 and January 2009, with a participation rate $(32 \cdot 2 \%)$ that corresponded to the theoretically expected rate upon which the sample size was calculated $^{(30)}$. Participants with missing data (i.e. at least two pages of the FFQ were uncompleted) or implausible dietary data (i.e. with values of nutrient intakes outside the 1 st and 99th percentiles of the distribution) were excluded ( $n$ 85). Those who reported currently being on diet for weight loss or for chronic CVD (diabetes, hypertension, dyslipidaemia) were also removed ( $n$ 195) from the analyses. Hence the final sample size used in the analysis was 1152 individuals.

The ORISCAV-LUX study received the approval of the Luxembourg national ethical committee and the national commission for private data protection. All participants provided written informed consent.

\section{Data collection}

Participant data were collected from three sources: (i) a self-administered questionnaire, including information on demographics, socio-economics, smoking history, diet and physical activity; (ii) anthropometric measurements; and (iii) blood sampling. Extensive quality control measures for the completeness and integrity of dietary and nondietary information were applied with the help of trained research nurses.

\section{Antbropometric measures and obesity assessment}

Height, body weight and waist circumference (WC) were measured in light clothing without shoes, according to previously published methods ${ }^{(2)}$. BMI was calculated as weight in kilograms divided by the square of height in metres $\left(\mathrm{kg} / \mathrm{m}^{2}\right)$. WC (centimetres) was measured at the level midway between the twelfth rib and the uppermost lateral border of the iliac crest at the end of normal expiration. Study participants were classified as normal weight $\left(\mathrm{BMI}<25 \cdot 0 \mathrm{~kg} / \mathrm{m}^{2}\right)$, overweight $(\mathrm{BMI} \geq 25 \cdot 0$ to $\left.<30.0 \mathrm{~kg} / \mathrm{m}^{2}\right)$ or obese $\left(\mathrm{BMI} \geq 30 \cdot 0 \mathrm{~kg} / \mathrm{m}^{2}\right)^{(31)}$. Global obesity was defined as BMI $\geq 30 \cdot 0 \mathrm{~kg} / \mathrm{m}^{2}$, while abdominal obesity was defined as $\mathrm{WC} \geq 102 \mathrm{~cm}$ for men and $\geq 88 \mathrm{~cm}$ for women ${ }^{(32)}$.

\section{Dietary intakes}

Dietary intake was assessed by means of a 134-item semiquantitative FFQ, self-administered and then verified with the participant by trained staff. The overall validity of the FFQ examined against nutritional biomarkers ${ }^{(33)}$ and a $3 \mathrm{~d}$ dietary record $^{(34)}$ showed a satisfactory performance in detecting and ranking micro- and macronutrients. Participants were required to assign the frequency and quantity of foods and beverages habitually consumed during the preceding 3 months. Food intakes were calculated by multiplying the self-reported food portion by the frequency of consumption. Energy and nutrient intake data were compiled using a French food composition table ${ }^{(35)}$. To account for energy intake, macronutrients were 
adjusted for total daily energy intake and expressed as percentages of total energy intake (\%E). Mathematically, a given intake was first divided by daily energy intake, then multiplied by 400 for protein and carbohydrate intake and by 900 for fat intake ${ }^{(36)}$.

\section{Animal protein sources}

Consistent with the guidelines of the US Department of Agriculture $^{(37)}$, protein derived from animal foods was divided into four broad categories: (i) meat; (ii) fish and shellfish; (iii) eggs; and (iv) milk products. The meat group was defined as the sum of the following types of meats: processed and unprocessed red meat including beef, pork, lamb, veal, game and poultry (chicken and turkey). Fish and shellfish included white fish, fatty fish such as salmon, canned fish such as tuna and seafood such as shrimp and squid. Milk products included milk, yoghurt and cheese (whole fat and reduced fat). The fraction of animal protein (8.59\%) derived from meat, fish and shellfish, eggs or dairy products mixed in prepared dishes (e.g. soup with pieces of meat, paella with meat or fish and shellfish, quiche with meat, eggs or cheese) was not included in the calculation of total animal protein.

\section{Potential confounding factors}

Based on an extensive literature review, several sociodemographic, lifestyle and dietary confounding factors were considered. Smoking status was dichotomized as 'non-smoker' and 'smoker'. Physical activity was calculated as the total amount of time engaged in physical activity per week. Education status was classified into primary, secondary or tertiary level. The dietary factors were total fat $(\mathrm{g} / \mathrm{d})$, total carbohydrate $(\mathrm{g} / \mathrm{d})$, total fibre $(\mathrm{g} / \mathrm{d})$ and fruit and vegetable intake $(\mathrm{g} / \mathrm{d})$. These variables have been described in detail elsewhere ${ }^{(29)}$.

\section{Statistical analysis}

Quantitative data were expressed as mean and standard deviation. Frequency tables were used for categorical findings. Participants' demographic and dietary characteristics according to global obesity (normal weight, overweight, obese) and abdominal obesity (absent, present) were compared by ANOVA or the $\chi^{2}$ test for contingency tables.

Total protein intake (animal and vegetal), total animal protein intake and protein intake from meats, fish and shellfish, eggs and milk products were used as the independent (explanatory) variables in the statistical analyses. All of these variables were expressed in $\mathrm{g} / \mathrm{d}$ and in percentage of total daily energy intake (\%E). Binary logistic regression was applied to assess the odds for global obesity (BMI $\geq 30 \cdot 0 \mathrm{~kg} / \mathrm{m}^{2}$ ) and for abdominal obesity (WC $\geq 102 \mathrm{~cm}$ for men and $\geq 88 \mathrm{~cm}$ for women), according to protein intakes. Three models were actually designed: model I adjusted for age and gender; model II further adjusted for education, smoking status, physical activity and intakes of total fat, carbohydrate, fibre, and fruit and vegetables; model III took also total vegetal protein into account. Finally, for each animal protein source, model III also made adjustment for protein intake from other animal sources. Results were expressed as odds ratios and 95\% confidence intervals. All main effect tests were two-sided at the $5 \%$ critical level $(P<0.05)$. Statistical calculations were done using the statistical software package IBM SPSS Statistics version 21 .

\section{Results}

\section{Participant characteristics according to animal protein intake}

Animal protein intake varied considerably according to gender $(P=0.039)$, with more men than women in each quartile of intake except the first. The intake of animal protein also varied significantly between normal-weight, overweight and obese participants $(P<0 \cdot 001)$; likewise in participants with abdominal obesity (Table 1 ).

\section{Protein intakes}

Participants with global or abdominal obesity exhibited higher total protein intake $(\% \mathrm{E})$ than those without the disorder (both $P<0 \cdot 001$; Table 2). Total animal protein intake and protein intakes from meat and from fish and shellfish all increased significantly with body weight status (all $P<0 \cdot 0001$; Table 3). Similarly, total animal protein intake, protein intake from meat and protein intake from fish and shellfish (all measured in $\mathrm{g} / \mathrm{d}$ or as \%E) were significantly higher in participants with abdominal obesity (all $P<0.01$ ). Protein derived from eggs or milk products did not differ according to body weight status.

\section{Multivariate modelling of global and abdominal obesity}

The major findings obtained from the multivariate modelling (models I-III) of global and abdominal obesity with respect to total protein, total animal protein and animal protein derived from each dietary source are displayed in Table 4. For model I (age- and gender-adjusted), total protein and total animal protein intakes (as \%E) were found to be associated with increased odds for both disorders (all $P<0 \cdot 001$ ). These associations remained significant when adjusting for education, smoking, physical activity and total fat, total carbohydrate, total fibre, fruit and vegetable intakes (model II).

When examining the individual dietary sources of animal protein and controlling for other animal protein sources, protein derived from meat was independently related to global obesity (model III: OR $=1 \cdot 18$; $95 \% \mathrm{CI}$ $1 \cdot 11,1.26)$ and abdominal obesity (OR $=1 \cdot 12 ; 95 \% \mathrm{CI}$ $1.05,1 \cdot 19)$. Likewise, a significant independent positive association was observed between protein derived from fish and shellfish and global obesity (model III: OR =1·18; 
Table 1 Participants' characteristics according to animal protein intake, Observation of Cardiovascular Risk Factors in Luxembourg (ORISCAV-LUX) study ( $n$ 1152)

\begin{tabular}{|c|c|c|c|c|c|c|c|c|c|}
\hline & \multicolumn{8}{|c|}{ Animal protein intake } & \multirow[b]{3}{*}{$P$ value* } \\
\hline & \multicolumn{2}{|c|}{$\begin{array}{c}\text { Quartile 1 } \\
(2 \cdot 26-8 \cdot 31 \% \mathrm{E})\end{array}$} & \multicolumn{2}{|c|}{$\begin{array}{c}\text { Quartile } 2 \\
(8.32-10.32 \% \mathrm{E})\end{array}$} & \multicolumn{2}{|c|}{$\begin{array}{c}\text { Quartile } 3 \\
(10 \cdot 32-12 \cdot 38 \% \mathrm{E})\end{array}$} & \multicolumn{2}{|c|}{$\begin{array}{c}\text { Quartile } 4 \\
(12 \cdot 39-25.37 \% \mathrm{E})\end{array}$} & \\
\hline & Mean & SD & Mean & SD & Mean & SD & Mean & SD & \\
\hline Age (years) & $45 \cdot 2$ & 13.8 & 43.4 & 13.4 & 43.6 & $12 \cdot 7$ & $44 \cdot 3$ & $12 \cdot 1$ & 0.38 \\
\hline \multirow[t]{2}{*}{ Physical activity (min/d) } & 207 & 199 & 193 & 191 & 178 & 172 & 200 & 177 & 0.30 \\
\hline & \multicolumn{2}{|c|}{$\%$} & \multicolumn{2}{|c|}{$\%$} & \multicolumn{2}{|c|}{$\%$} & \multicolumn{2}{|c|}{$\%$} & \\
\hline Gender & & & & & & & & & 0.039 \\
\hline Male ( $n$ 578) & \multirow{2}{*}{\multicolumn{2}{|c|}{$43 \cdot 1$}} & \multicolumn{2}{|c|}{$51 \cdot 1$} & \multicolumn{2}{|c|}{$54 \cdot 6$} & \multicolumn{2}{|c|}{$52 \cdot 1$} & \\
\hline Female ( $n$ 574) & & & \multicolumn{2}{|c|}{48.9} & \multicolumn{2}{|c|}{$45 \cdot 4$} & \multicolumn{2}{|c|}{47.9} & \\
\hline Education level & & & & & & & & & 0.21 \\
\hline Primary (n 302) & \multicolumn{2}{|c|}{$28 \cdot 0$} & \multicolumn{2}{|c|}{$23 \cdot 0$} & \multicolumn{2}{|c|}{$25 \cdot 6$} & \multicolumn{2}{|c|}{$29 \cdot 2$} & \\
\hline Secondary (n 532) & \multicolumn{2}{|c|}{$44 \cdot 7$} & \multirow{2}{*}{\multicolumn{2}{|c|}{$\begin{array}{l}53.5 \\
23.4\end{array}$}} & \multicolumn{2}{|c|}{$44 \cdot 8$} & \multicolumn{2}{|c|}{$43 \cdot 3$} & \\
\hline Tertiary ( $n$ 305) & \multicolumn{2}{|c|}{$27 \cdot 3$} & & & \multicolumn{2}{|c|}{29.5} & \multicolumn{2}{|c|}{27.4} & \\
\hline Smoking status & & & & & & & & & 0.25 \\
\hline Non-smoker (n 903) & \multicolumn{2}{|c|}{$78 \cdot 1$} & \multicolumn{2}{|c|}{$81 \cdot 0$} & \multicolumn{2}{|c|}{$74 \cdot 6$} & & & \\
\hline Current smoker (n 249) & & & & & & & & & \\
\hline Global obesity & & & & & & & & & $<0.001$ \\
\hline Normal weight ( $n$ 546) & & & & & & & & & \\
\hline Overweight $(n 375)$ & & & & & & & & & \\
\hline Obese (n 231) & & & & & & & & & \\
\hline Abdominal obesity (n 330) & & & & & & & & & $<0.001$ \\
\hline
\end{tabular}

$\% \mathrm{E}$, percentage of total daily energy intake.

${ }^{*} P$ values are from ANOVA (continuous variables) or the $x^{2}$ test (categorical variables).

Table 2 Participants' total energy and macronutrient intakes according to global and abdominal obesity, Observation of Cardiovascular Risk Factors in Luxembourg (ORISCAV-LUX) study ( $n$ 1152)

\begin{tabular}{|c|c|c|c|c|c|c|c|c|c|c|c|c|}
\hline & \multicolumn{6}{|c|}{ Global obesity } & \multirow[b]{3}{*}{$P$ value } & \multicolumn{4}{|c|}{ Abdominal obesity } & \multirow[b]{3}{*}{$P$ value } \\
\hline & \multicolumn{2}{|c|}{$\begin{array}{l}\text { Normal weight } \\
\quad(n 546)\end{array}$} & \multicolumn{2}{|c|}{$\begin{array}{l}\text { Overweight } \\
\quad(n 375)\end{array}$} & \multicolumn{2}{|c|}{$\begin{array}{l}\text { Obese } \\
(n 231)\end{array}$} & & \multicolumn{2}{|c|}{$\begin{array}{c}\text { No } \\
(n 822)\end{array}$} & \multicolumn{2}{|c|}{$\begin{array}{c}\text { Yes } \\
(n 330)\end{array}$} & \\
\hline & Mean & SD & Mean & SD & Mean & SD & & Mean & SD & Mean & SD & \\
\hline Age (years) & 39.9 & 12.9 & $46 \cdot 3$ & $12 \cdot 3$ & $50 \cdot 3$ & $11 \cdot 3$ & $<0.001$ & 41.5 & $12 \cdot 8$ & $50 \cdot 6$ & $11 \cdot 3$ & $<0.001$ \\
\hline Physical activity (min/week) & 760 & 941 & 764 & 930 & 740 & 928 & 0.95 & 783 & 966 & 694 & 847 & 0.15 \\
\hline Weight (kg) & $64 \cdot 7$ & $9 \cdot 6$ & $79 \cdot 1$ & $9 \cdot 2$ & $96 \cdot 7$ & $14 \cdot 0$ & $<0.001$ & $70 \cdot 2$ & $12 \cdot 2$ & $89 \cdot 6$ & $16 \cdot 5$ & $<0.001$ \\
\hline Total energy (MJ/d) & $10 \cdot 33$ & 0.38 & $9 \cdot 92$ & 0.38 & $10 \cdot 38$ & 0.42 & 0.22 & $10 \cdot 28$ & 0.38 & $10 \cdot 00$ & 0.41 & $0.28^{*}$ \\
\hline Total energy (kcal/d) & 2468 & 907 & 2370 & 920 & 2480 & 1009 & 0.22 & 2458 & 913 & 2391 & 980 & $0 \cdot 28^{*}$ \\
\hline Total protein $(\mathrm{g} / \mathrm{d})$ & $93 \cdot 0$ & $36 \cdot 2$ & 92.9 & $36 \cdot 3$ & $100 \cdot 5$ & $38 \cdot 8$ & 0.021 & 93.9 & 35.9 & 95.9 & $39 \cdot 0$ & 0.40 \\
\hline Total protein (\%E) & $15 \cdot 3$ & $3 \cdot 0$ & $15 \cdot 9$ & 3.0 & $16 \cdot 8$ & $3 \cdot 2$ & $<0.001$ & $15 \cdot 5$ & 3.0 & $16 \cdot 4$ & $3 \cdot 3$ & $<0.001$ \\
\hline Total carbohydrate $(\mathrm{g} / \mathrm{d})$ & 266.5 & $108 \cdot 8$ & $250 \cdot 4$ & $103 \cdot 9$ & $255 \cdot 8$ & $111 \cdot 3$ & 0.07 & $264 \cdot 1$ & $107 \cdot 7$ & $246 \cdot 6$ & $107 \cdot 5$ & 0.013 \\
\hline Total carbohydrate (\%E) & $43 \cdot 2$ & $7 \cdot 4$ & $42 \cdot 6$ & 7.5 & 41.4 & 6.7 & 0.008 & $43 \cdot 2$ & 7.4 & $41 \cdot 2$ & 6.9 & $<0.001$ \\
\hline Total fat $(g / d)$ & $107 \cdot 7$ & $46 \cdot 5$ & $99 \cdot 3$ & $41 \cdot 8$ & $105 \cdot 1$ & $48 \cdot 6$ & 0.024 & $104 \cdot 8$ & $44 \cdot 8$ & $103 \cdot 6$ & $47 \cdot 5$ & 0.70 \\
\hline Total fat (\%E) & $39 \cdot 1$ & $7 \cdot 0$ & $37 \cdot 7$ & $7 \cdot 0$ & $38 \cdot 1$ & $7 \cdot 0$ & 0.012 & $38 \cdot 2$ & $6 \cdot 8$ & 39.0 & $7 \cdot 4$ & 0.07 \\
\hline Saturated fat $(g / d)$ & $37 \cdot 7$ & $17 \cdot 2$ & $35 \cdot 3$ & $17 \cdot 6$ & $37 \cdot 2$ & $18 \cdot 8$ & 0.14 & 36.9 & $17 \cdot 5$ & $36 \cdot 5$ & $18 \cdot 1$ & 0.68 \\
\hline Saturated fat (\%E) & $13 \cdot 6$ & 2.7 & $13 \cdot 2$ & 3.0 & $13 \cdot 3$ & 2.9 & 0.06 & $13 \cdot 3$ & $2 \cdot 8$ & $13 \cdot 6$ & $3 \cdot 0$ & 0.21 \\
\hline Alcohol $(\mathrm{g} / \mathrm{d})$ & $7 \cdot 5$ & $10 \cdot 0$ & $10 \cdot 6$ & $12 \cdot 8$ & 9.4 & $12 \cdot 6$ & $<0.001$ & $9 \cdot 1$ & 11.5 & $8 \cdot 3$ & $11 \cdot 6$ & 0.31 \\
\hline Total fibre $(\mathrm{g} / \mathrm{d})$ & $25 \cdot 3$ & $10 \cdot 3$ & $24 \cdot 0$ & $10 \cdot 4$ & $25 \cdot 2$ & $10 \cdot 6$ & 0.18 & 24.9 & $10 \cdot 3$ & 24.9 & $10 \cdot 7$ & 0.99 \\
\hline
\end{tabular}

$\% \mathrm{E}$, percentage of total daily energy intake.

*Total energy intake was positively correlated with waist circumference (as a continuous variable).

$95 \%$ CI $1.05,1.33)$ and abdominal obesity (model III: $\mathrm{OR}=1 \cdot 17 ; 95 \% \mathrm{CI} 1 \cdot 04,1 \cdot 30)$. Protein consumption from eggs and milk products was unrelated to global and abdominal obesity.

\section{Discussion}

The present study has demonstrated a positive independent association between animal protein intake and both global and abdominal obesity in presumably healthy adults in Luxembourg. Higher animal protein intake was associated with higher odds of global and abdominal obesity, specifically from meat (red meat, poultry) and fish and shellfish consumption, but not eggs or milk products. The odds of global obesity increased by $18 \%$ for every $1 \%$ increase in total energy intake derived from meat products or from fish and shellfish (both $P<0 \cdot 01$ ). Our results indicate that the four selected sources of animal protein 
Table 3 Participants' total vegetal and animal protein intakes and protein intakes from main animal sources, according to global and abdominal obesity status, Observation of Cardiovascular Risk Factors in Luxembourg (ORISCAV-LUX) study $(n 1152)$

\begin{tabular}{|c|c|c|c|c|c|c|c|c|c|c|c|c|}
\hline & \multicolumn{6}{|c|}{ Global obesity } & \multirow[b]{3}{*}{$P$ value } & \multicolumn{4}{|c|}{ Abdominal obesity } & \multirow[b]{3}{*}{$P$ value } \\
\hline & \multicolumn{2}{|c|}{$\begin{array}{l}\text { Normal weight } \\
\quad(n 546)\end{array}$} & \multicolumn{2}{|c|}{$\begin{array}{l}\text { Overweight } \\
(n \text { 375) }\end{array}$} & \multicolumn{2}{|c|}{$\begin{array}{l}\text { Obese } \\
(n 231)\end{array}$} & & \multicolumn{2}{|c|}{$\begin{array}{c}\text { No } \\
(n \text { 822) }\end{array}$} & \multicolumn{2}{|c|}{$\begin{array}{c}\text { Yes } \\
(n 330)\end{array}$} & \\
\hline & Mean & SD & Mean & SD & Mean & SD & & Mean & SD & Mean & SD & \\
\hline \multicolumn{13}{|l|}{ Protein source } \\
\hline Vegetal protein $(\mathrm{g} / \mathrm{d})$ & $29 \cdot 8$ & $12 \cdot 1$ & $28 \cdot 0$ & $11 \cdot 3$ & $30 \cdot 1$ & $13 \cdot 3$ & 0.040 & 29.5 & $11 \cdot 8$ & $28 \cdot 7$ & $12 \cdot 9$ & 0.31 \\
\hline Vegetal protein (\%E) & 4.9 & 1.1 & 4.8 & 1.2 & 4.9 & $1 \cdot 1$ & 0.69 & 4.9 & $1 \cdot 1$ & $5 \cdot 0$ & $1 \cdot 2$ & 0.77 \\
\hline Animal protein $(\mathrm{g} / \mathrm{d})$ & $60 \cdot 9$ & 28.0 & 61.8 & 28.4 & $67 \cdot 8$ & $29 \cdot 2$ & 0.007 & $61 \cdot 8$ & 28.0 & 64.6 & 29.5 & 0.13 \\
\hline Animal protein (\%E) & $10 \cdot 0$ & 3.0 & $10 \cdot 6$ & $3 \cdot 3$ & 11.4 & $3 \cdot 3$ & $<0.001$ & $10 \cdot 2$ & $3 \cdot 1$ & $11 \cdot 1$ & $3 \cdot 3$ & $<0.001$ \\
\hline \multicolumn{13}{|l|}{ Animal-protein food sources } \\
\hline Protein from meat $(\mathrm{g} / \mathrm{d})$ & $28 \cdot 6$ & $20 \cdot 8$ & $29 \cdot 1$ & 19.5 & 34.9 & $24 \cdot 0$ & 0.001 & 29.6 & 21.4 & $31 \cdot 1$ & $20 \cdot 7$ & 0.30 \\
\hline Protein from meat (\%E) & 4.6 & 2.5 & $5 \cdot 0$ & 2.5 & $5 \cdot 6$ & $2 \cdot 9$ & $<0.001$ & 4.8 & 2.6 & $5 \cdot 3$ & $2 \cdot 7$ & 0.008 \\
\hline Protein from fish and shellfish $(\mathrm{g} / \mathrm{d})$ & $8 \cdot 7$ & $7 \cdot 0$ & 9.8 & $7 \cdot 9$ & 11.4 & $12 \cdot 7$ & $<0.001$ & $9 \cdot 1$ & $7 \cdot 3$ & $10 \cdot 9$ & $11 \cdot 5$ & 0.001 \\
\hline Protein from fish and shellfish (\%E) & 1.5 & $1 \cdot 2$ & $1 \cdot 8$ & 1.4 & 1.9 & 1.6 & $<0.001$ & 1.6 & $1 \cdot 3$ & 1.9 & 1.5 & $<0.001$ \\
\hline Protein from eggs $(\mathrm{g} / \mathrm{d})$ & 1.7 & $2 \cdot 4$ & 1.5 & 1.5 & 1.6 & 1.6 & 0.34 & 1.6 & $2 \cdot 2$ & 1.6 & 1.6 & 0.78 \\
\hline Protein from eggs (\%E) & 0.3 & 0.4 & 0.3 & 0.2 & 0.3 & 0.3 & 0.56 & 0.3 & 0.3 & 0.3 & 0.3 & 0.44 \\
\hline Protein from milk products $(\mathrm{g} / \mathrm{d})$ & 14.9 & $11 \cdot 8$ & 14.5 & $12 \cdot 7$ & 14.4 & $10 \cdot 6$ & 0.84 & $14 \cdot 6$ & $12 \cdot 1$ & 14.9 & $11 \cdot 1$ & 0.70 \\
\hline Protein from milk products (\%E) & 2.5 & 1.7 & 2.4 & 1.7 & 2.4 & 1.7 & 0.90 & $2 \cdot 4$ & 1.7 & $2 \cdot 6$ & 1.7 & 0.15 \\
\hline
\end{tabular}

$\% \mathrm{E}$, percentage of total daily energy intake.

Table 4 Multivariate modelling (models I-III) of global and abdominal obesity with respect to intakes of total protein, total animal protein and protein from main dietary sources based on 1152 participants from the Observation of Cardiovascular Risk Factors in Luxembourg (ORISCAV-LUX) study

\begin{tabular}{|c|c|c|c|c|c|c|}
\hline \multirow[b]{2}{*}{ Protein variable } & \multicolumn{3}{|c|}{ Global obesity } & \multicolumn{3}{|c|}{ Abdominal obesity } \\
\hline & OR & $95 \% \mathrm{Cl}$ & $P$ value & OR & $95 \% \mathrm{Cl}$ & $P$ value \\
\hline \multicolumn{7}{|c|}{ Total protein (\%E) } \\
\hline Model I & $1 \cdot 15$ & $1.09,1.20$ & $<0.001$ & 1.12 & $1.07,1.17$ & $<0.001$ \\
\hline Model II & $1 \cdot 18$ & $1 \cdot 12,1.25$ & $<0.001$ & 1.14 & $1.08,1.20$ & $<0.001$ \\
\hline \multicolumn{7}{|c|}{ Total animal protein (\%E) } \\
\hline Model I & $1 \cdot 12$ & $1.07,1.17$ & $<0.001$ & 1.11 & $1.06,1.16$ & $<0.001$ \\
\hline Model II & $1 \cdot 15$ & $1.09,1.22$ & $<0.001$ & $1 \cdot 12$ & $1 \cdot 07,1 \cdot 18$ & $<0.001$ \\
\hline Model IIla & $1 \cdot 18$ & $1.11,1.25$ & $<0.001$ & $1 \cdot 13$ & $1.07,1.19$ & $<0.001$ \\
\hline \multicolumn{7}{|c|}{ Protein from meat $(\% \mathrm{E})$} \\
\hline Model I & $1 \cdot 15$ & $1.09,1.22$ & $<0.001$ & 1.13 & $1.07,1.19$ & $<0.001$ \\
\hline Model II & $1 \cdot 16$ & $1.09,1.24$ & $<0.001$ & 1.12 & $1.05,1.18$ & $<0.001$ \\
\hline Model IIIb & $1 \cdot 18$ & $1.11,1.26$ & $<0.001$ & $1 \cdot 12$ & $1.05,1.19$ & $<0.001$ \\
\hline \multicolumn{7}{|c|}{ Protein from fish and shellfish (\%E) } \\
\hline Model I & $1 \cdot 13$ & $1.02,1.25$ & 0.020 & $1 \cdot 13$ & $1.02,1.24$ & 0.016 \\
\hline Model II & $1 \cdot 16$ & $1.04,1.30$ & 0.009 & 1.16 & $1.04,1.29$ & 0.016 \\
\hline Model IIIb & $1 \cdot 18$ & $1.05,1.33$ & 0.004 & 1.17 & $1.04,1.30$ & 0.007 \\
\hline \multicolumn{7}{|c|}{ Protein from eggs (\%E) } \\
\hline Model I & $1 \cdot 18$ & $0.74,1.88$ & 0.50 & 1.14 & $0.74,1.75$ & 0.57 \\
\hline Model II & $1 \cdot 11$ & $0.66,1.85$ & 0.70 & 0.88 & $0.54,1.45$ & 0.62 \\
\hline Model Illb & $1 \cdot 11$ & $0.66,1.87$ & 0.69 & 0.84 & $0.51,1.40$ & 0.51 \\
\hline \multicolumn{7}{|c|}{ Protein from milk products $(\% \mathrm{E})$} \\
\hline Model I & 0.99 & $0.90,1.08$ & 0.80 & 0.99 & $0.91,1.07$ & 0.77 \\
\hline Model II & 1.03 & $0.94,1.14$ & 0.51 & 1.03 & $0.94,1.12$ & 0.56 \\
\hline Model IIlb & 1.08 & $0.98,1.19$ & 0.13 & 1.05 & $0.96,1.15$ & 0.30 \\
\hline
\end{tabular}

$\% \mathrm{E}$, percentage of total daily energy intake.

Model I: adjusted for age and gender.

Model II: adjusted for age, gender, education, smoking, physical activity and intakes of total fat, total carbohydrates, total fibre, and fruit and vegetables.

Model IIla: adjusted for covariates in model II and total vegetal protein (\%E).

Model IIIb: adjusted for covariates in model II, total vegetal protein (\%E) and protein from other animal sources (\%E).

may have a differential effect on obesity, independent of age, gender, education level, lifestyle behaviours and other dietary factors.

Although the literature on the animal protein intakeobesity relationship is not abundant, our data are consistent with a recent cross-sectional study conducted in a Belgian population ${ }^{(17)}$, which showed positive associations between animal protein intake and BMI and WC in males, but not in females. To further investigate our data, a separate gender-specific sensitivity analyses showed similar positive significant relationships for both men and women with animal protein. These findings are also in line 
with a recently published prospective study, the Chicago Western Electric Study ${ }^{(15)}$, which examined the association between protein intake and obesity in over 1000 American men over 7 years. Animal protein intake was positively associated with overweight and obesity, independent of energy, carbohydrate and fat intakes. Both abovementioned studies $^{(15,17)}$ also found an inverse association between vegetable protein intake and obesity; no such associations were observed in the present study. We believe we have added to the current literature by evaluating the relationship between specific animal-protein food sources and obesity. The present findings concur with positive associations observed in other cross-sectional studies between red meat consumption and $\mathrm{BMI}^{(25,38,39)}, \mathrm{WC}^{(25,40)}$ and the metabolic syndrome ${ }^{(40,41)}$. Total meat consumption has also been positively associated with weight gain in both normalweight and overweight adults over a 5-year follow-up period in a large European cohort ${ }^{(27)}$

Although a consensus exists that energy restriction promotes weight loss, the effect of varying the macronutrient composition of the diet (fat, protein, carbohydrates) on weight loss has been debated ${ }^{(42)}$. Several intervention trials indicate that low-carbohydrate diets promote a greater degree of weight loss in the short term than a conventional high-carbohydrate, low-fat diet ${ }^{(43-45)}$, even when energy intake is matched $^{(46)}$, but over the long term weight loss is similar to other that achieved with energy-restricted dietary patterns ${ }^{(47)}$. Other studies have demonstrated that highprotein diets with concomitant decreases in energy intake may result in sustained weight loss or improved health ${ }^{(48,49)}$. In high-protein diets, weight loss is initially high due to fluid loss related to reduced carbohydrate intake, overall energy restriction and ketosis-induced appetite suppression ${ }^{(42)}$.

The biological mechanisms that might explain the adverse relationship between animal protein intake and the risk of obesity are still unclear. The high cholesterol, Fe and C-reactive protein levels in red meat may have detrimental effects on body weight and health outcomes ${ }^{(40)}$. In addition, high-protein foods of animal sources (in particular red meats) are energy-dense, high-fat foods, particularly rich in SFA. This macronutrient composition may contribute to the adverse effect on body weight and energy regulation. Energy density may be a key element in body-weight regulation as it may alter appetite control signals (i.e. hunger and satiety) ${ }^{(50)}$.

Most of the randomized controlled trials investigating protein consumption on health outcomes have focused on adjusting total protein in relation to other macronutrients in the diet, rather than on the types of protein or specific sources. As recognized by others ${ }^{(6)}$, few randomized controlled trials examining protein intake and health outcomes have reported protein sources (animal $v$. vegetable). Including different food sources of protein within a high- or low-protein diet may contribute to the conflicting results among studies regarding the animal protein-obesity association. The present findings confirm our initial hypothesis regarding the varying effects according to animal dietary sources. The nutritional content of different protein sources included in the diet may have different influences on body weight and therefore help to explain some of the disparate findings. This will be an important distinction to make in future dietary trials. In addition, the diversity of study designs (cross-sectional, longitudinal, clinical trials) and differential control for confounders may explain the inconsistent findings.

Our contemporary nationwide database ${ }^{(2,30)}$ constitutes an opportunity to examine associations between animal protein and anthropometric measures, with a focus on different animal protein sources, namely meat, fish, eggs and dairy products. As the ORISCAV-LUX measured a large set of potential dietary and non-dietary confounders, we trust that our 'European' findings contribute to a growing body of evidence indicating that high intakes of meat may not have a favourable effect on body composition. As in similar population-based studies, the ORISCAV-LUX survey has some limitations, related mainly to the current absence of a gold standard for dietary assessment. Food group and nutrient intakes were estimated by self-reported data. It is well known that overweight/obese people under-report their dietary intake to a greater extent than normal-weight people ${ }^{(51)}$. Despite intensive efforts to minimize dietary reporting inaccuracies through extensive control procedures $^{(2)}$, diet is a complex exposure factor with measurement being subject to imprecision and a wide range of errors and biases. Two extensive validation studies ${ }^{(33,34)}$ have been performed to examined the performance of the FFQ, showing that it performed well in assessing intakes of several foods and micronutrients and the observed correlations were within the range noted by other investigators. However, the complexity and expense to perform $\mathrm{N}$ analyses in $24 \mathrm{~h}$ urine collections (gold standard recovery biomarker to validate protein intake) may constitute a drawback in the present study.

The cross-sectional design of the study did not allow conclusions on causal relationships to be made. It precludes inferences about long-term dietary effects on obesity. However, it is less plausible that obese participants have altered their diet by consuming more meats and fish, since those who are currently on diet to reduce their weight or for a cardiovascular health problem (diabetes, hypertension, lipid disorders) were excluded from the analyses. The fact that there was no difference in physical activity between those who were normal weight and those who were obese (the same goes for abdominal obesity) suggests that the majority of those who were obese are trying to resist the state that they are in and perhaps may explain why they are eating more protein-rich foods and less carbohydrates. Unfortunately, we were not able to distinguish the individual associations between particular types of meat (red meat or poultry), as the FFQ was unable to capture this difference. The meat group included a wide range of meats, such as pork, beef, lamb and poultry, and cold processed meats, such as salami. Similarly, a variety of fish types were 
included within 'fish and shellfish' and distinctions between, for example, fatty fish and canned fish were unable to be made due to few cases.

Meat intake can be linked to adverse effects on adiposity through plausible mechanisms and the results from other previous prospective studies lend support to our findings. Along with the effect of meat consumption on the risk of other diseases, such as CVD, diabetes, metabolic syndrome and colorectal cancer, our findings build an added argument against adopting a high-animal-protein diet, specifically from meat, to maintain healthy weight. These findings may have practical implications for public health dietary recommendations. Notwithstanding, it should be kept in mind that protein is one of the three major macronutrients and an important source of energy, needed for both younger and elderly age groups ${ }^{(52)}$. In the present studied population, total protein intake contributed $15 \cdot 8 \%$ to total energy intake. Two-thirds of total protein intake (mean intake of $94.5 \mathrm{~g} / \mathrm{d}$ ) was derived from animal protein (mean intake of $62.6 \mathrm{~g} / \mathrm{d}$ ) and one-third was from vegetable protein (mean intake of $29.3 \mathrm{~g} / \mathrm{d}$; data not shown).

\section{Conclusion}

Consumption of animal protein, particularly that derived from meat products, showed a positive association with adiposity measures among presumably healthy adults in Luxembourg, independently of gender, age, educational level, smoking status, physical activity and intakes of fibre, fat and carbohydrates. The consumption of meat, a major source of animal protein, plays a vital role in providing a diversified and nutritious diet. Animal products are major sources of a wide range of essential micronutrients; in particular, vitamin $\mathrm{A}$ and minerals such as Fe and $\mathrm{Zn}$. Any emphasis placed on the need to reduce animal protein intake in the diet of apparently healthy people should be seen in the sense of opting for other sources of animal protein, such as eggs or dairy products, rather than increasing carbohydrate or fat consumption. In the scarcity of robust evidence from long-term, high-cost prospective and interventional trials, our findings may constitute a relevant contribution to the prevention and control of obesity.

\section{Acknowledgements}

Financial support: This research work was supported by a research grant from the National Fund of Research (Fond National de Recherche (FNR)); project DIQUA-LUX, 5870 404. The FNR had no role in the design, analysis or writing of this article. Conflict of interest: None. Author contributions: Both M.G. and G.E.C. contributed equally to the supervision. A. Alkerwi was involved in the conception and design of the ORISCAV-LUX survey, coordinated the field data collection, conceived the present research, contributed to data analyses and drafted the manuscript. N.S. conducted the statistical analyses and contributed to data interpretation. A.-F.D. participated in the statistical analyses. J.D.B. and A. Albert contributed to the critical revision of the manuscript and intellectual content. G.E.C. and M.G. provided expertise and oversight throughout the process. All of the authors reviewed drafts and approved the final version of the manuscript. Ethics of buman subject participation: The ORISCAV-LUX study received the approval of the Luxembourg national ethical committee and the national commission for private data protection. All participants provided written informed consent.

\section{References}

1. World Health Organization (2003) Diet, Nutrition and the Prevention of Chronic Diseases. WHO Technical Report Series no. 916. Geneva: WHO.

2. Alkerwi A, Sauvageot N, Donneau AF et al. (2010) First nationwide survey on cardiovascular risk factors in GrandDuchy of Luxembourg (ORISCAV-LUX). BMC Public Health 10, 468

3. Astrup A (2001) Healthy lifestyles in Europe: prevention of obesity and type II diabetes by diet and physical activity. Public Health Nutr 4, 499-515.

4. Ledikwe JH, Blanck HM, Kettel Khan L et al. (2006) Dietary energy density is associated with energy intake and weight status in US adults. Am J Clin Nutr 83, 1362-1368.

5. Mendoza JA, Drewnowski A \& Christakis DA (2007) Dietary energy density is associated with obesity and the metabolic syndrome in US adults. Diabetes Care 30, 974-979.

6. Santesso N, Akl EA, Bianchi M et al. (2012) Effects of higherversus lower-protein diets on health outcomes: a systematic review and meta-analysis. Eur J Clin Nutr 66, 780-788.

7. Schwingshackl L \& Hoffmann G (2013) Long-term effects of low-fat diets either low or high in protein on cardiovascular and metabolic risk factors: a systematic review and metaanalysis. Nutr J 12, 48

8. Wycherley TP, Moran LJ, Clifton PM et al. (2012) Effects of energy-restricted high-protein, low-fat compared with standard-protein, low-fat diets: a meta-analysis of randomized controlled trials. Am J Clin Nutr 96, 1281-1298.

9. Hite AH, Feinman RD, Guzman GE et al. (2010) In the face of contradictory evidence: report of the Dietary Guidelines for Americans Committee. Nutrition 26, 915-924.

10. Paddon-Jones D, Westman E, Mattes RD et al. (2008) Protein, weight management, and satiety. Am J Clin Nutr 87, issue $5,1558 \mathrm{~S}-1561 \mathrm{~S}$.

11. Farnsworth E, Luscombe ND, Noakes M et al. (2003) Effect of a high-protein, energy-restricted diet on body composition, glycemic control, and lipid concentrations in overweight and obese hyperinsulinemic men and women. $\mathrm{Am} \mathrm{J}$ Clin Nutr 78, 31-39.

12. Merchant AT, Anand SS, Vuksan V et al. (2005) Protein intake is inversely associated with abdominal obesity in a multi-ethnic population. J Nutr 135, 1196-1201.

13. Velasquez MT \& Bhathena SJ (2007) Role of dietary soy protein in obesity. Int J Med Sci $\mathbf{4}, 72-82$.

14. Ashton EL, Dalais FS \& Ball MJ (2000) Effect of meat replacement by tofu on CHD risk factors including copper induced LDL oxidation. J Am Coll Nutr 19, 761-767.

15. Bujnowski D, Xun P, Daviglus ML et al. (2011) Longitudinal association between animal and vegetable protein intake and obesity among men in the United States: the Chicago Western Electric Study. J Am Diet Assoc 111, 1150-1155.e1. 
16. Kelemen LE, Kushi LH, Jacobs DR Jr et al. (2005) Associations of dietary protein with disease and mortality in a prospective study of postmenopausal women. Am J Epidemiol 161, 239-249.

17. Lin Y, Bolca S, Vandevijvere S et al. (2011) Plant and animal protein intake and its association with overweight and obesity among the Belgian population. Br J Nutr 105, 1106-1116.

18. Elliott P, Stamler J, Dyer AR et al. (2006) Association between protein intake and blood pressure: the INTERMAP Study. Arch Intern Med 166, 79-87.

19. Tzoulaki I, Brown IJ, Chan Q et al. (2008) Relation of iron and red meat intake to blood pressure: cross sectional epidemiological study. BMJ 337, a258.

20. Song Y, Manson JE, Buring JE et al. (2004) A prospective study of red meat consumption and type 2 diabetes in middle-aged and elderly women: the Women's Health Study. Diabetes Care 27, 2108-2115.

21. Djousse L, Gaziano JM, Buring JE et al. (2009) Egg consumption and risk of type 2 diabetes in men and women. Diabetes Care 32, 295-300.

22. Pan A, Sun Q, Bernstein AM et al. (2013) Changes in red meat consumption and subsequent risk of type 2 diabetes mellitus three cohorts of US men and women. JAMA Intern Med 173, 1328-1335.

23. Chen GC, Lv DB, Pang Z et al. (2013) Red and processed meat consumption and risk of stroke: a meta-analysis of prospective cohort studies. Eur J Clin Nutr 67, 91-95.

24. Wagemakers JJ, Prynne CJ, Stephen AM et al. (2009) Consumption of red or processed meat does not predict risk factors for coronary heart disease; results from a cohort of British adults in 1989 and 1999. Eur J Clin Nutr 63 , 303-311.

25. Wang Y \& Beydoun MA (2009) Meat consumption is associated with obesity and central obesity among US adults. Int $J$ Obes (Lond) 33, 621-628.

26. Xu F, Yin XM \& Tong SL (2007) Association between excess bodyweight and intake of red meat and vegetables among urban and rural adult Chinese in Nanjing, China. Asia Pac J Public Health 19, 3-9.

27. Vergnaud AC, Norat T, Romaguera D et al. (2010) Meat consumption and prospective weight change in participants of the EPIC-PANACEA study. Am J Clin Nutr 92, 398-407.

28. Clifton P (2012) Effects of a high protein diet on body weight and comorbidities associated with obesity. Br J Nutr 108, Suppl. 2, S122-S129.

29. Alkerwi A, Donneau AF, Sauvageot N et al. (2012) Dietary, behavioural and socio-economic determinants of the metabolic syndrome among adults in Luxembourg: findings from the ORISCAV-LUX study. Public Health Nutr 15, 849-859.

30. Alkerwi A, Sauvageot N, Couffignal S et al. (2010) Comparison of participants and non-participants to the ORISCAV-LUX population-based study on cardiovascular risk factors in Luxembourg. BMC Med Res Methodol 10, 80.

31. Chu NF, Rimm EB, Wang DJ et al. (1998) Clustering of cardiovascular disease risk factors among obese schoolchildren: the Taipei Children Heart Study. Am J Clin Nutr 67, 1141-1146.

32. National Cholesterol Education Program (NCEP) Expert Panel on Detection, Evaluation, and Treatment of High Blood Cholesterol in Adults (Adult Treatment Panel III) (2002) Third Report of the National Cholesterol Education Program (NCEP) Expert Panel on Detection, Evaluation, and Treatment of High Blood Cholesterol in Adults (Adult Treatment Panel III) final report. Circulation 106, 3143-3421.

33. Sauvageot N, Alkerwi A, Albert A et al. (2013) Use of food frequency questionnaire to assess relationships between dietary habits and cardiovascular risk factors in NESCAV study: validation with biomarkers. Nutr J 12, 143.
34. Sauvageot N, Alkerwi Aa, Adelin A et al. (2013) Validation of the food frequency questionnaire used to assess the association between dietary habits and cardiovascular risk factors in the NESCAV Study. J Nutr Food Sci 3, 208.

35. Hercberg S, Arnault N, Astorg P et al. (2006) Tables de composition des aliments/SU.VI.MAX. Paris: Economica.

36. Willett WC, Howe GR \& Kushi LH (1997) Adjustment for total energy intake in epidemiologic studies. Am J Clin Nutr 65, 4 Suppl, 1220S-1228S

37. US Department of Agriculture, Agricultural Research Service (2010) Report of the Dietary Guidelines Advisory Committee on the Dietary Guidelines for Americans. Washington, DC: USDA/ARS.

38. Okubo H, Sasaki S, Murakami K et al.; Freshmen in Dietetic Courses Study II group (2008) Three major dietary patterns are all independently related to the risk of obesity among 3760 Japanese women aged 18-20 years. Int J Obes (Lond) 32, $541-549$.

39. Maskarinec G, Novotny R \& Tasaki K (2000) Dietary patterns are associated with body mass index in multiethnic women. J Nutr 130, 3068-3072.

40. Azadbakht L \& Esmaillzadeh A (2009) Red meat intake is associated with metabolic syndrome and the plasma C-reactive protein concentration in women. J Nutr 139, 335-339.

41. Babio N, Sorli M, Bullo M et al. (2012) Association between red meat consumption and metabolic syndrome in a Mediterranean population at high cardiovascular risk: crosssectional and 1-year follow-up assessment. Nutr Metab Cardiovasc Dis 22, 200-207.

42. St Jeor ST, Howard BV, Prewitt TE et al.; Nutrition Committee of the Council on Nutrition Physical Activity, and Metabolism of the American Heart Association (2001) Dietary protein and weight reduction: a statement for healthcare professionals from the Nutrition Committee of the Council on Nutrition, Physical Activity, and Metabolism of the American Heart Association. Circulation 104, 1869-1874.

43. Foster GD, Wyatt HR, Hill JO et al. (2003) A randomized trial of a low-carbohydrate diet for obesity. $N$ Engl J Med $\mathbf{3 4 8}$, 2082-2090.

44. Samaha FF, Iqbal N, Seshadri P et al. (2003) A lowcarbohydrate as compared with a low-fat diet in severe obesity. N Engl J Med 348, 2074-2081.

45. Stern L, Iqbal N, Seshadri P et al. (2004) The effects of lowcarbohydrate versus conventional weight loss diets in severely obese adults: one-year follow-up of a randomized trial. Ann Intern Med 140, 778-785.

46. Halyburton AK, Brinkworth GD, Wilson CJ et al. (2007) Low- and high-carbohydrate weight-loss diets have similar effects on mood but not cognitive performance. Am J Clin Nutr 86, 580-587.

47. Brinkworth GD, Noakes M, Buckley JD et al. (2009) Longterm effects of a very-low-carbohydrate weight loss diet compared with an isocaloric low-fat diet after $12 \mathrm{mo} . \mathrm{Am} \mathrm{J}$ Clin Nutr 90, 23-32.

48. Halton TL \& Hu FB (2004) The effects of high protein diets on thermogenesis, satiety and weight loss: a critical review. J Am Coll Nutr 23, 373-385.

49. Skov AR, Toubro S, Ronn B et al. (1999) Randomized trial on protein vs carbohydrate in ad libitum fat reduced diet for the treatment of obesity. Int J Obes Relat Metab Disord 23, 528-536.

50. Vergnaud AC, Estaquio C, Czernichow S et al. (2009) Energy density and 6-year anthropometric changes in a middleaged adult cohort. Br J Nutr 102, 302-309.

51. Macdiarmid J \& Blundell J (1998) Assessing dietary intake: who, what and why of under-reporting. Nutr Res Rev 11, 231-253.

52. Chernoff R (2004) Protein and older adults. J Am Coll Nutr 23, 6 Suppl. 627S-630S. 